\title{
Modeling and simulation of fixed bed regenerators for a multi- tower decoupled advanced solar combined cycle
}

\author{
Iván Mesonero Jesús Febres Susana López \\ IK4-TEKNIKER, Spain, \{ivan.mesonero, jesus.febres, susana.lopez\}@tekniker.es
}

\begin{abstract}
Two dynamic models of fixed bed regenerators for metallic and ceramic configurations have been developed in Modelica. These models have been both worked out within CAPTURE European project and will serve as design tool for a fixed bed regenerative heat exchange system. The present article describes in detail both models and presents a case study that compares experimental and simulation results for the testing of a ceramic honeycomb regenerative matrix.
\end{abstract}

Keywords: fixed bed regenerator, ceramic honeycomb, stacked wire cloths, solar Brayton cycle

\section{Introduction}

The recently granted EU R\&D project CAPTURE (http://www.capture-solar-energy.eu) pursues a new concept of central receiver system based on the Decoupled Solar Combined Cycle (DSCC) plant concept (see Figure 1). In such a plant, a multi-tower approach is employed with a solar Brayton cycle turbine on the top of each tower.

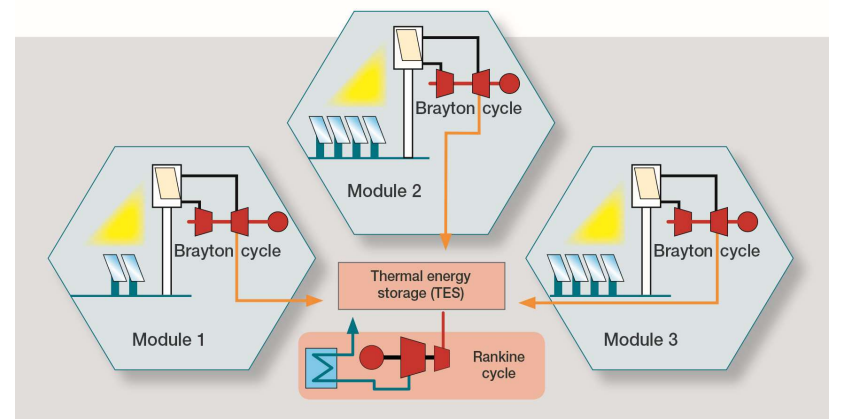

Figure 1. CAPTURE plant configuration based on DSCC concept

In CAPTURE project, a non-pressurized volumetric receiver will be employed to feed the solar turbine using a fixed bed regenerative heat exchange system for connecting both pressurized and non-pressurized air loops (see Figure 2). The fixed bed regenerative heat exchangers are alternatively connected to the two different air loops through a group of two-way on-off valves. Thus, the system allows the continuous operation of the receiver and the turbine through the charging and discharging of a certain number of fixed bed regenerators.

Two approaches have been defined and modelled for the configuration of the fixed bed regenerator matrix material, a metallic approach based on stacked wire cloths, and a ceramic approach based on ceramic honeycombs monoliths. For the analysis of both options, one-dimension dynamic Modelica model of each approach have been developed within CAPTURE project and will be included in a free Modelica library. These models had to be parametric and flexible enough to allow the analysis of the effect of the design variables, such as the material characteristics and the bed geometry, in the behavior of the regenerative heat exchange.

Besides the two presented models, Modelica models of the receiver and the turbine shall be developed within CAPTURE in order to completely simulate the plant shown in Figure 2. This development will be carried out during incoming phase of the project.

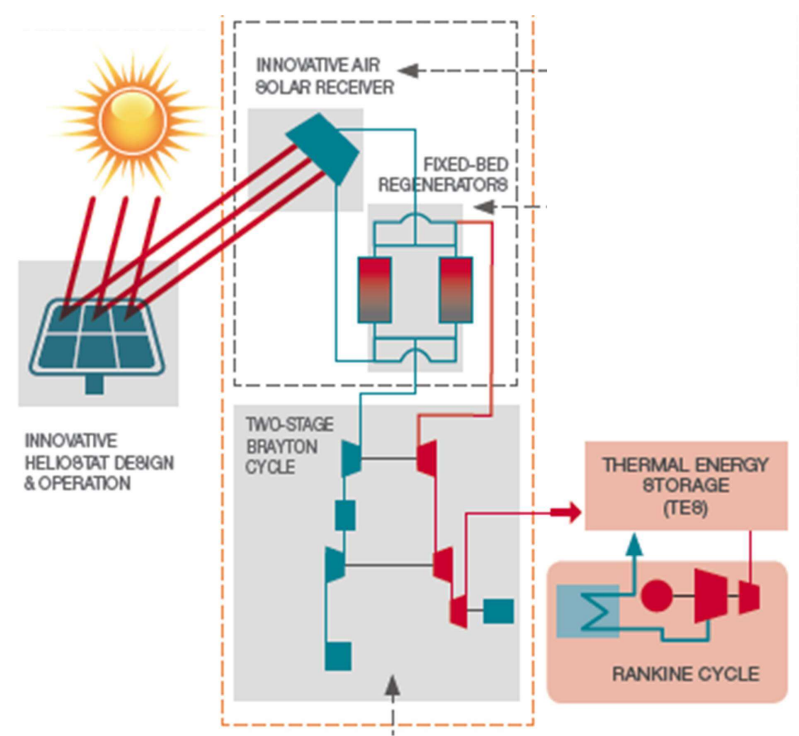

Figure 2. Main subsystems of a single module of CAPTURE plant 


\section{Metallic regenerative bed model}

A cylindrical regenerative bed has been modelled (MetalicRegenerativeBed1D) considering the following main assumptions:

- Regenerative beds are made by a randomly stacked woven-screen matrix with plain square (see Figure $3)$.

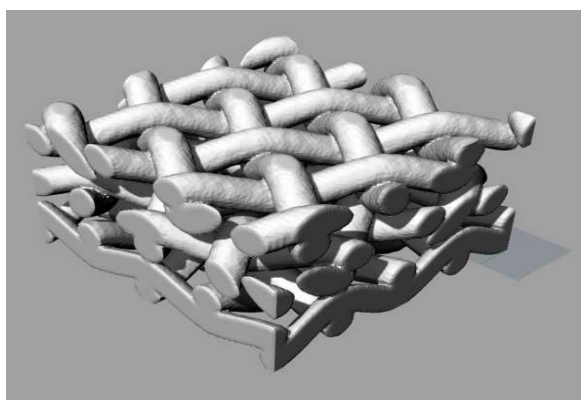

Figure 3. 3D view of three randomly stacked screens (plain square weave)

- The matrix is made of metallic materials and the model takes into account the dependency of their conductivity and specific heat capacity with the temperature.

- One-dimensional fluid flow is assumed, including only as heat transfer phenomenon the heat convection between the fluid and the matrix, i.e. radiative heat transfer is disregarded.

- One-dimensional heat conduction along the matrix (parallel to the fluid flow) is assumed. Perfect insulation is considered at the lateral area, thus heat losses can only be taken into account through upper and lower end of the matrix

\subsection{Model structure}

The model is mainly composed of two components that represent the solid and the fluid phases of the regenerative bed.

The model also includes a replaceable porosity function for the calculation of the volumetric porosity ${ }^{1}$ of the matrix (T), which is required for further calculation in equations (5) - (10). The following options are available to be chosen from a drop down menu: ESDUPorosity, NASAPorosity, ArmourCannonPorosity and XuWirtzPorosity. All of them were described by ( $\mathrm{Li}$ $\&$ Peterson, 2006). However, some discrepancies were found for the porosity calculation defined by $\mathrm{Xu} \&$ Wirtz, in consequence the original reference $(\mathrm{Xu} \&$ Wirtz, 2002) was chosen for this case in order to define the necessary equations implemented in the code.

\footnotetext{
${ }^{1}$ In here the volumetric porosity is defined as the ratio between the void volume and the total volume of a porous body.
}

The following equations (1, 2, 3 and 4) describe how to calculate the porosity in each option.

- ESDU:

$$
\text { T }=1-0.25 * \pi * \frac{d_{w}}{p i t c h}
$$

- NASA:

$$
\text { T }=1-0.25 * \pi * f_{\text {crimping }} * \frac{d_{w}}{\text { pitch }}
$$

- Armour \& Cannon:

$$
\begin{aligned}
& \text { T }=1-\pi *\left(\frac{A * B}{2 *(A+B)}\right) * \sqrt{1+\left(\frac{A}{1+A}\right)^{2}} \\
& A=\frac{d_{w}}{\text { pitc } w} \\
& B=\frac{d_{w}}{t}
\end{aligned}
$$

- Xu \& Wirtz:

$$
\begin{aligned}
& \text { T }=1+\frac{3.906 E^{-4} * \pi * C *\left(\frac{d w}{p i t c h}\right)}{C_{f}} \\
& C_{f}=\frac{L}{n_{\text {layer }} * 2 * d_{w}} \\
& C=123 *\left(\frac{d_{w}}{\text { pitch }}\right)^{4}-384 *\left(\frac{d_{w}}{\text { pitch }}\right)^{2}-640
\end{aligned}
$$

Where:

- $d_{w}$ is the wire diameter;

- pitch is the distance between two wires or the aperture;

- $f_{\text {crimping }}$ is a factor that describes how compressed are the meshes in the matrix;

- $t$ is the thickness of the mesh;

- $L$ is the regenerative bed length;

- $n_{\text {layer }}$ is the number of layers in the matrix.

It must be noted that for the last two options the user must provide the value of some more parameters, the thickness of the wire mesh or the number of layers in the matrix, as shown in Figure 4. 


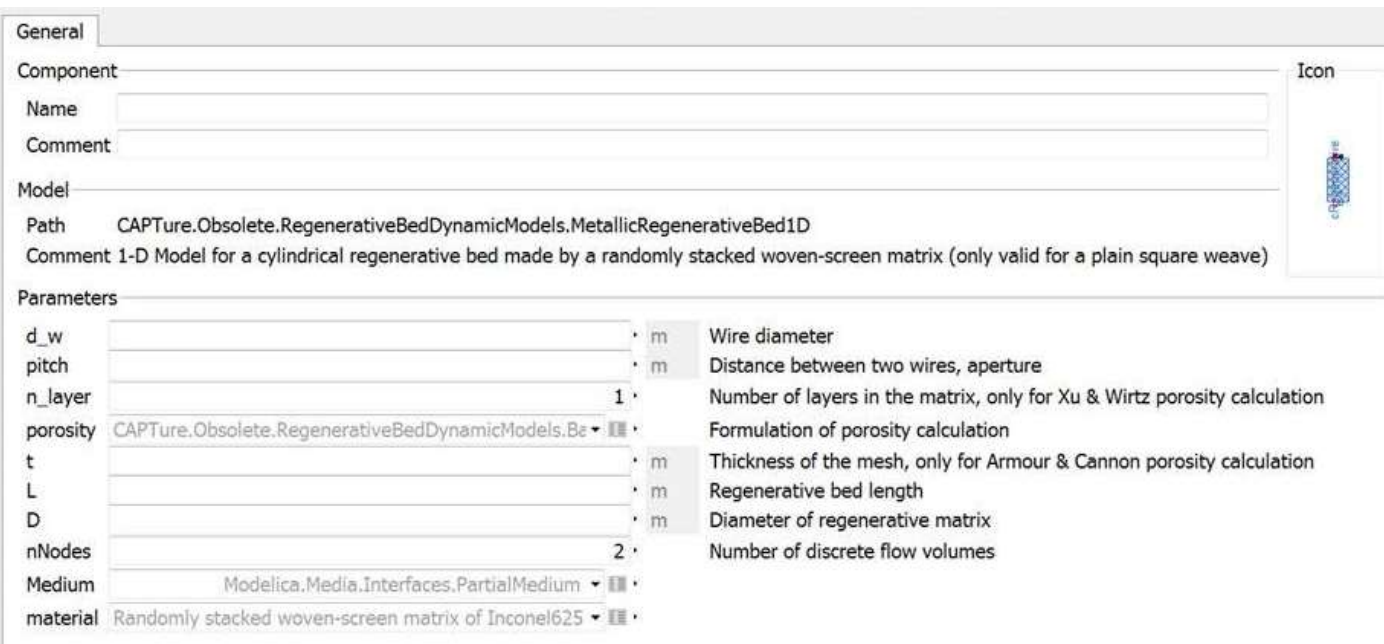

Figure 4. Parameter dialog for the metallic regenerator model

\subsubsection{Solid phase}

Solid phase component is an instance of the class HollowCyliner that is a lumped parameter thermal system in the radial direction (there is no radial variation of the solid temperature). It is composed of an array of nodes which are instances of the class HollowCylinder_Lumped that represents one section of the solid material along its axis. Each node consists of two classes. One that describes the conduction along the material, and other that represents the thermal inertia of the material section.

In order to calculate the thermal characteristics of the solid phase model, the material properties have to be entered in the model. HollowCylinder class includes a replaceable instance of a class that contains the characteristics of the material of the regenerative bed. This material can be selected in the "Solid" tab of the main model. In addition, the regenerative bed total mass can be entered as a parameter. If no value of the total mass is used, it is calculated multiplying the material density by the total volume of the solid.

If a new material is required, the material class has to be declared as a Modelica package extended from the base class PartialMaterial. This package must contain the thermal and physical properties of the chosen material. The minimal set of properties required consists of the density, the thermal conductivity and the specific heat capacity. All of them may be defined either as a constant or as function of the temperature.

Since the model was meant to describe the behavior of porous materials and more in concrete woven-screen matrixes, this class takes into account the porosity of the matrix and the conductivities of the solid and the fluid in order to calculate the real thermal conductivity of the matrix using the following equation (Martini, 2004):

$$
=k_{\text {gas }} *\left(\frac{\left(\frac{1+\left(\frac{k_{\text {material }}}{k_{\text {mas }}}\right)}{1-\left(\frac{k_{\text {material }}}{k_{\text {gas }}}\right)}\right)-(1-\mathbb{\tau})}{\left(\frac{1+\left(\frac{k_{\text {material }}}{k_{\text {gas }}}\right)}{1-\left(\frac{k_{\text {material }}}{k_{\text {gas }}}\right)}\right)+(1-\mathbb{\tau})}\right)
$$

Where:

- $k_{\text {matrix }}$ is the thermal conductivity of the matrix;

- $k_{g a s}$ is the thermal conductivity of the gas in the matrix;

- $k_{\text {material }}$ is the thermal conductivity of the matrix material (solid).

The class defining the solid phase has an array of inputs named thermalconductivity_medium in order to have access to the instantaneous value of the internal variable of the fluid phase fluidPhase.heatTransfer.lambdas that is exactly the instantaneous value of the thermal conductivity of the gas in the different nodes along the matrix.

\subsubsection{Matrix materials}

Apart from the basic partial models described in the previous sections, four specific materials have been added to the Materials library: DIN EN 10095, DIN 17742, DIN 17470 and DIN EN 10302. These materials were chosen taking into account the expected operating temperatures, their availability as meshed material, thermal and mechanical properties, and sintering possibility. In all cases, the specific heat capacity and thermal conductivity are temperature dependent values while density is assumed constant. In 
addition, whether the material is sintered or not is not taken into account in this version of the model.

However, the effect of sintering the material shall be included in further versions as it influences the thermal conductivity (Li \& Peterson, 2006) and the mechanical stability during the cycling regime, e.g. sintering stabilizes an in-line stacked bed (unstable before sintering) as a permanent link between wire meshes is guaranteed.

Regarding this two last properties, the available information from datasheets was fitted to polynomial expressions (linear or quadratic) in most cases and logarithmic expressions in others.

\subsubsection{Fluid phase}

Fluid phase component is an instance of the class DynamicRegenerativeBedFluidPhase that is based on the DynamicPipe class from the Modelica Standard Library (Casella, 2009) that is the model of a straight pipe with distributed mass, energy and momentum balances providing the complete balance equations for one-dimensional fluid flow. It treats the partial differential equations with the finite volume method and a staggered grid scheme for momentum balances.

The main differences between the original DynamicPipe and the DynamicRegenerativeBedFluidPhase are the following:

- Specific equations have been implemented under the Detailed option of FlowModel. When the Detailed option is selected, the relationship between the mass flow rate and the pressure loss is determined with experimental correlation for a flow through an infinite randomly stacked woven-screen matrix.

- Flow friction characteristics were originally defined by Kays \& London (Kays \& London, 1998). They determined experimentally the relationship between the friction factor and the Reynolds number for different porosity values of the matrix. But the equations implemented within this model correspond to the following approximation determined by Martini (Martini 2004):

$$
\begin{aligned}
& d p=\frac{m_{\text {flow }}^{2} * L * C_{w}}{2 * A^{2} * \rho *\left(\frac{D_{h}}{4}\right)} \\
& \log C_{w}= \\
& \left\{\begin{array}{l}
1.73-0.93 * \log R e \quad \text { if } R e<60 \\
0.714-0.365 * \log \operatorname{Re} \text { if } 60 \leq \operatorname{Re}<1000 \\
0.015-0.125 * \log \operatorname{Re} \text { if } 1000 \leq \operatorname{Re}
\end{array}\right.
\end{aligned}
$$

Where:

- $d p$ is the pressure drop along the matrix;

- $m_{\text {flow }}$ is the fluid mass flow rate;

- $L$ is the length of the matrix;

- $C_{w}$ is the factor of friction for matrix;

- $A$ is the area of flow;

- $\rho$ is the density of the fluid at regenerator;

- $D_{h}$ is the hydraulic diameter of the matrix;

- $R e$ is the Reynolds number.

- A new option was added to the list of classes that describe the convective heat transfer within this model with equation (7). It is especially suited for gas flow through an infinite randomly stacked woven-screen matrix being a correlation from Organ (Organ, 2010) of experimental data from wire screens and crossed rods simulating wire screens from Kays \& London (Kays \& London, 1998).

- Main assumptions of the correlation are: perfect stacking, i.e. screens touching is assumed, and volumetric porosity between 0.602 and 0.832 .

$$
S t * \sqrt{\operatorname{Pr}^{3}}=\frac{1.25}{\sqrt{R e}}
$$

Where:

- $\quad S t$ is the Stanton number;

- $\operatorname{Pr}$ is the Prandtl number;

- $R e$ is the Reynolds number.

Regarding the parameterization of the fluid phase model, the hydraulic radius, $r_{h}$, of the individual wire screen and matrixes is determined by equations (8) and (9) when porosity is calculated by the expression defined by ESDU (Organ, 1997) (Kays \& London, 1998):

$$
\begin{aligned}
& r_{h}=\frac{\text { pitch }}{\pi}-\frac{d_{w}}{4} \\
& r_{h}=\frac{d_{w} * \mathbb{T}}{4 *(1-\Phi)}
\end{aligned}
$$

It is worth to mention also that for both correlations (flow friction and convective heat transfer) the Reynolds 
number in equation (10) is calculated with a velocity $\left(\mathrm{v}_{\mathrm{s}}\right)$ that is not the real velocity of the fluid along the matrix.

$$
\begin{aligned}
R e & =\frac{\rho * v_{S} * D_{h}}{\mu} \\
& =\frac{\frac{m_{\text {flow }}}{A_{c}} * D_{h}}{\mu} \\
& =\frac{\frac{m_{\text {flow }}}{A_{\text {fr }} * D_{h}}}{\mu}
\end{aligned}
$$

Where:

- $\quad \rho$ is the density of the fluid at the matrix;

- $D_{h}$ is the hydraulic diameter of the matrix;

- $\mu$ is the cinematic viscosity of the fluid;

- $m_{\text {flow }}$ is the fluid mass flow rate;

- $A_{C}$ is the free flow area of the matrix;

- $A_{f r}$ is the frontal area of the matrix;

- $\mathbb{T}$ is the volumetric porosity of the matrix.

The reason for that is that the free flow area is calculated as the product of the frontal area of the matrix and its volumetric porosity. Usually, the volumetric porosity and the screen porosity have different values, being the second one bigger that the first one. Accordingly, the computed values for the fluid velocity within the fluid phase model will be bigger than the real ones.

\section{Ceramic regenerative bed model}

During the specification definition phase of CAPTURE, the partners decided that the type of ceramic regenerator to be modelled was to be a honeycomb with straight channels (see Figure 5).

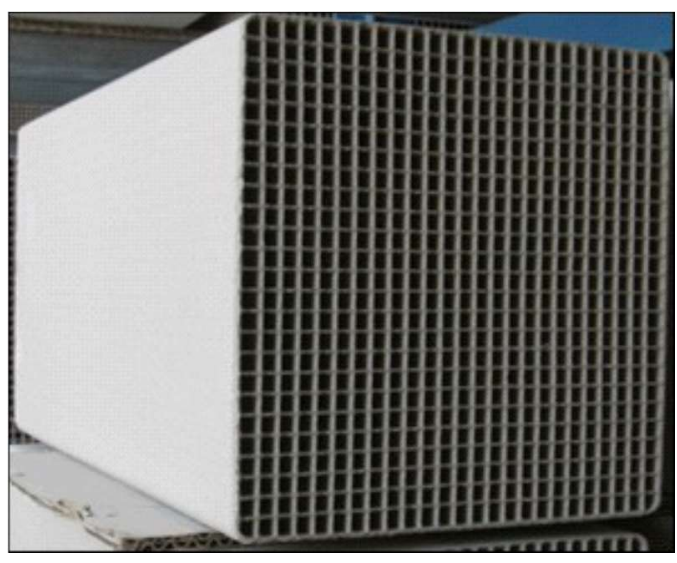

Figure 5. Cordierite honeycomb brick with high density of straight channels.

This model (CeramicRegenerativeBed1D) is based on the modelling approach presented by Muske et al (Muske, 2000). Even though it was originally meant for checkerwork regenerators, the model may be used for other regenerators with the same type of geometry, i.e. parallel straight channel geometry (two dimensions honeycomb). Each channel of the regenerator is modelled as a hollow cylinder tube, whose external wall is assumed perfectly isolated. The radius of the fluid channel, the internal radius $r_{i}$, is one-half of the average hydraulic diameter of the real fluid channels and the outside radius is given by equation (11):

$$
r_{o}=\sqrt{\frac{m}{\pi * \rho * N_{c} * L}+\frac{D_{h}^{2}}{4}}
$$

Where:

- $N_{C}$ is the total number of gas channels;

- $D_{h}$ is the hydraulic diameter of the gas channel;

- $m$ is the total mass of the bed;

- $\quad \rho$ is the density of the bed material;

- $L$ is the length of the bed.

The following considerations were taken into account when modelling the ceramic regenerative bed:

- The fluid velocity in the tubes is determined assuming a uniform distributed fluid flow through all channels.

- As in the metallic bed, the ceramic regenerative bed model is constituted by two namely solid and fluid phases.

- Regarding the solid phase, there is no radial variation of the temperature (lumped parameter model in the radial direction is assumed).

Note that for simulations where cycling regimes are required, the last assumption is expected to be valid only when the cycle time of the system is, at least, an order of magnitude bigger than the characteristic time for radial heat conduction in the material (Muske 2010) which is defined by:

$$
\tau=\frac{\left(r_{o}-r_{i}\right)^{2}}{\alpha}
$$

Where:

- $\tau$ is the characteristic time for radial heat conduction;

- $\quad \alpha$ is the thermal diffusivity of the bed material;

- $r_{i}$ is the radius of the gas channel in the tube;

- $r_{o}$ is the outside radius of the tube.

For the case of highly channeled honeycombs, the reduced thickness of walls assures a good agreement with the last assumption.

\subsection{Model structure}

Figure 6 shows the icon of the Modelica model of the ceramic regenerator bed. Both heat and fluid ports are taken directly from the Modelica Standard Library, which means the model is compatible with any element found in Modelica.Fluid and Modelica.Thermal. 


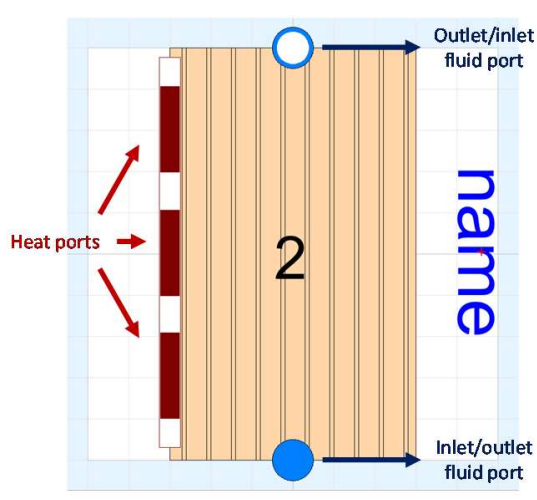

Figure 6. Modelica ceramic regenerative bed model icon. The model allows users to initialize the temperature of the fluid and the solid elements. Initial values can be input in the "Initialization" tab. If no value is passed to the model, $24{ }^{\circ} \mathrm{C}$ is used as default value for both temperatures.

In order to define the geometry of the regenerative bed, six parameters can be input in the "General" tab:

- The bed length: length in [m];

- The bed cross-section area: area_s in $\left[\mathrm{m}^{2}\right]$;

- Channels cross-section area: area_c in $\left[\mathrm{m}^{2}\right]$;

- Channels cross-section perimeter: perimeter_c in [m];

- Number of channels: N_c ;

- Segmentation perpendicular to heat conduction: nNodes.

Note that the model is discretised in finite volumes (solid and fluid volumes) and the degree of discretisation is defined by the nNodes parameter.

As mentioned previously, the model is composed, as can be appreciated in Figure 7, by two components that represent the solid and the fluid phases of the regenerative bed.

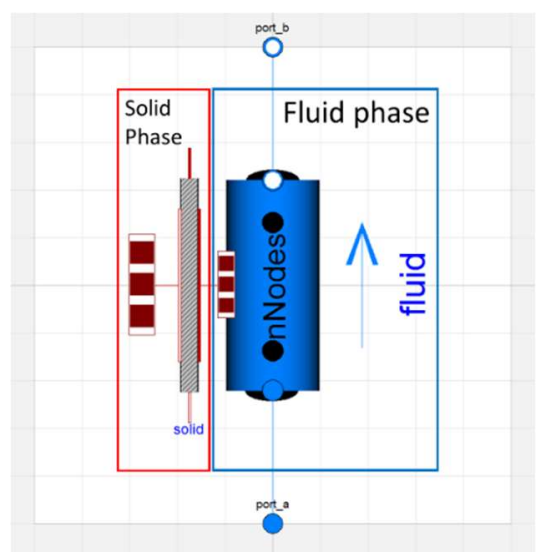

Figure 7. Diagram of the ceramic regenerative bed model.

\subsubsection{Solid phase}

The model presented in section 2.1.1 was used to model the solid phase of the ceramic regenerator as this model allows the user to work with non-porous material. There is a Boolean parameter in the "Porosity" tab that disables the use of the equations mentioned in section 2.1.1., making the model suitable to represent ceramic materials.

\subsubsection{Fluid phase}

The fluid phase component is an instance of the DynamicPipe class from the Modelica Standard Library which is the model of a straight pipe with distributed mass, energy and momentum balances providing the complete balance equations for onedimensional fluid flow. It treats the partial differential equations with the finite volume method and a staggered grid scheme for momentum balances.

Most of the parameters that define the DynamicPipe have been fixed and only three of them (the fluid medium, the heat transfer model and the flow model) are accessible from the GUI.

A new option was added to the list of classes for the heat transfer that describes the convective heat transfer with a correlation for rough pipes by Bhatti and Shah (Muske 2000).

\section{Ceramic regenerative bed case study}

The case study proposed in this paper is centered on the ceramic honeycomb approach for regenerative matrix. The proposed model was verified against the experimental results presented in a technical report elaborated by SANDERS Associates in 1980 (Sanders, 1980). This report describes the application of the regenerator in a solar prototype small plant as well as the experimental set-up and test results of a ceramic honeycomb regenerator manufactured for real demonstration at laboratory level. Next paragraphs describe the manufactured regenerator and the tests performed that were be employed for the case study.

\subsection{Ceramic regenerator description}

The ceramic regenerator is a cylindrical matrix installed inside an internally insulated cylindrical pressure vessel, the complete system is called Thermal Storage Module or TSM. Dimensions of the complete matrix are $914 \mathrm{~mm}$ (36 inches) in diameter and $787 \mathrm{~mm}$ (31 inches) in length. Base material for the regenerator matrix are cylindrical ceramic honeycombs logs from CORNING with the same long as the complete matrix and a diameter of $114 \mathrm{~mm}$ ( 4.5 inches). The shape of the logs 
are modified in order to get the sectors that finally conforms the complete regenerative cylinder. Figure 8, Figure 9 and Figure 10 clarify above description.

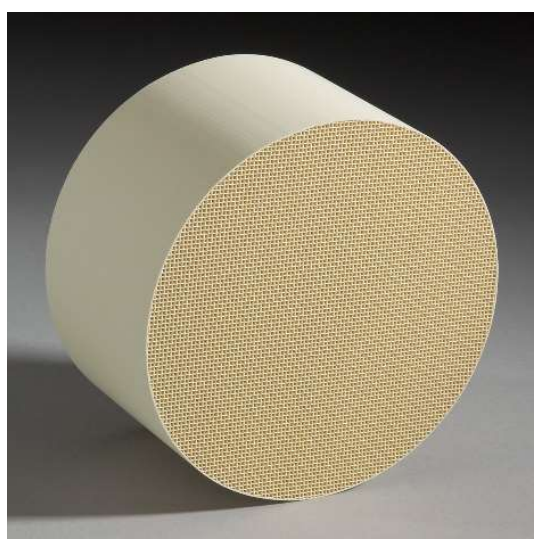

Figure 8. Typical Cordierite honeycomb cylinder for catalytic converters in automotive application from CORNING

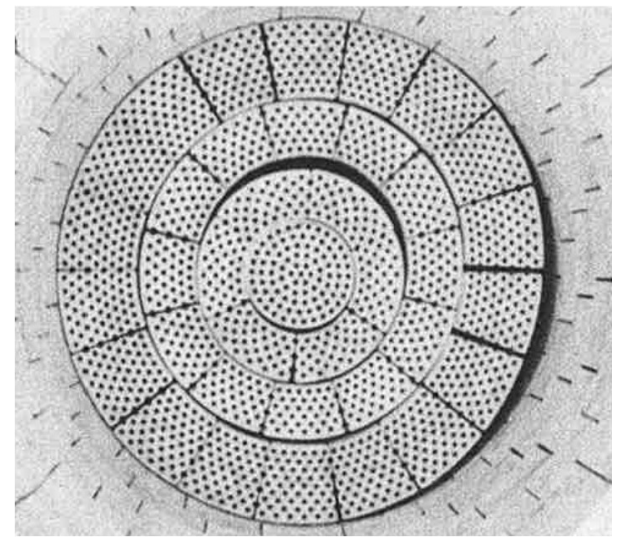

Figure 9. Picture of the internal cross section of a ceramic heat exchanger with the same manufacturing approach as SANDERS's regenerator (Sheindlin, 1986)

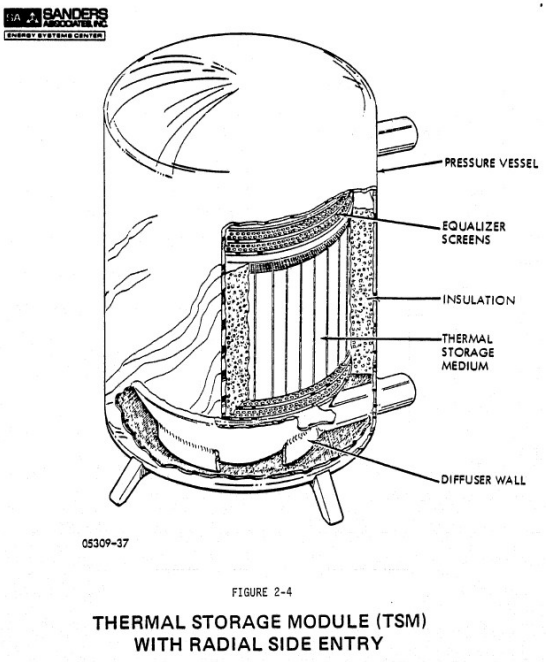

Figure 10. Diagram of the Thermal Storage Module developed by SANDERS (Sanders, 1980)
The Cordierite log employed for manufacturing the TSM is a square cell based honeycomb designated by $300 / 12$, which corresponds to $300 \mathrm{cpsi}$ (cells per square inch) and 12×10-3 inches of wall thickness. The thermal properties of the Cordierite material in the honeycomb are summarized in Table 1

Table 1. Thermal properties of the Cordierite material in the honeycomb

\begin{tabular}{|l|l|l|l|l|}
\hline $\begin{array}{l}\text { Temperature } \\
{ }^{\circ} \mathrm{C}\left({ }^{\circ} \mathrm{F}\right)\end{array}$ & $\begin{array}{l}260 \\
(500)\end{array}$ & $\begin{array}{l}399 \\
(750)\end{array}$ & $\begin{array}{l}538 \\
(1000)\end{array}$ & $\begin{array}{l}815 \\
(1500)\end{array}$ \\
\hline $\begin{array}{l}\text { Specific heat } \\
\mathrm{J} / \mathrm{kg} \mathrm{K} \\
\left(\mathrm{BTU} / \mathrm{lb}{ }^{\circ} \mathrm{F}\right)\end{array}$ & $\begin{array}{l}1005 \\
(0.24)\end{array}$ & $\begin{array}{l}1118 \\
(0.267)\end{array}$ & $\begin{array}{l}1193 \\
(0.285)\end{array}$ & $\begin{array}{l}1289 \\
(0.308)\end{array}$ \\
\hline $\begin{array}{l}\text { Thermal } \\
\text { conductivity } \\
\text { W/mK (BTU } \\
\left.\text { in/h ft2 }{ }^{\circ} \mathrm{F}\right)\end{array}$ & $1.44(10)$ constant \\
\hline $\begin{array}{l}\mathrm{Bulk} \text { density } \\
\mathrm{g} / \mathrm{cm} 3(\mathrm{lb} / \mathrm{ft} 3)\end{array}$ & \multicolumn{4}{|l}{$0.589(36.4)$ constant } \\
\hline
\end{tabular}

\subsection{Test set-up and instrumentation}

The test set-up is mainly composed of a ceramic regenerator, a four-way valve, a gas burner, a compressor, control valves and piping. The test schematic can be seen in Figure 11.

The test set-up is equipped with sensors allowing the analysis of the regenerator performance under different test conditions. Principal sensors of interest for the analysis are: air mass flow meter, air temperature sensors, air pressure sensors and temperature sensors for the measurement of the ceramic material in different positions along the bed.

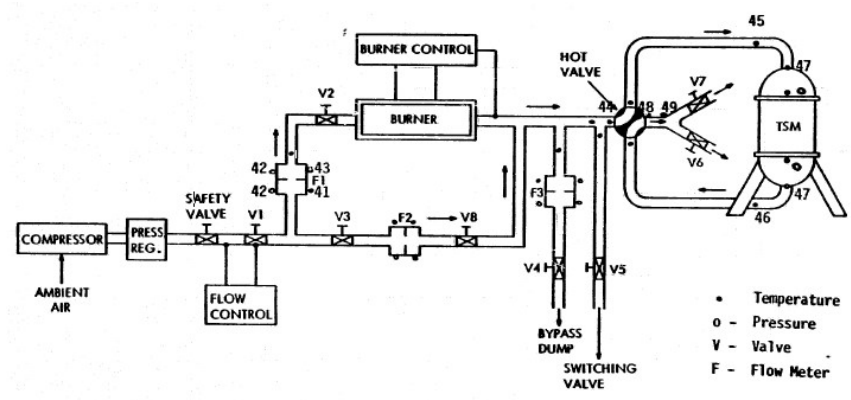

Figure 11. Test set-up schematic developed by SANDERS (Sanders, 1980)

\subsection{Test selection for the case study}

For the model validation two different types of tests were chosen, a single shot test and a cycling test. Both tests are described in the next paragraphs. 


\subsubsection{Single shot test}

This test is based on a sudden heating up (or cooling down) of the regenerator with an air stream that flows from top to bottom (or from bottom to top). The initial temperature of the entire matrix is constant and homogeneous.

The objective of the test is to analyze the thermal performance of the bed in terms of thermocline propagation along the matrix (transient response).

Table 2. Selected single shot test data

\begin{tabular}{|l|l|}
\hline Air flow direction & Upward \\
\hline Air mass flow kg/s $(\mathrm{lb} / \mathrm{s})$ & $0.19(0.43)$ \\
\hline Air temperature ${ }^{\circ} \mathrm{C}\left({ }^{\circ} \mathrm{F}\right)$ & $702(1295)$ \\
\hline $\begin{array}{l}\text { Initial homogeneous temperature in } \\
\text { the matrix }{ }^{\circ} \mathrm{C}\left({ }^{\circ} \mathrm{F}\right)\end{array}$ & $146(295)$ \\
\hline
\end{tabular}

Figure 12 shows the original graph with the experimental results of the selected test (Sanders 1980) and the simulation results obtained overlapped. The simulation results were obtained with the model described on section 3 under the general test conditions of Table 2, but it has not been possible to accurately reproduce the variable inlet temperature of the experimental data. Moreover, the initial temperature of the entire regenerative bed was assumed homogeneous but the report points out the existence of an initial temperature profile along the bed due to experimental difficulties in setting up initial test conditions. Consequently, further analysis of the system transient shall be performed in order to understand the quantitative deviations. Nevertheless, a good agreement, from the qualitative point of view, has been achieved between the experimental and the simulation results.

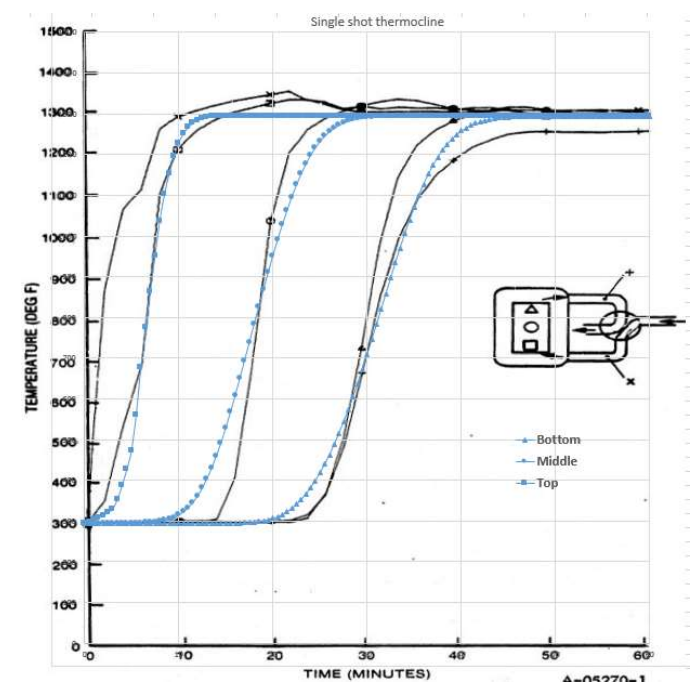

Figure 12. Experimental (black) and simulation (blue) results of single shot test of the ceramic regenerative bed model.

\subsubsection{Cycling test}

This test is based on a continuous cyclic operation (charging and discharging) of the regenerator starting from an initial steady state (constant temperature within the entire matrix). During the charging phase, hot air flows at atmospheric pressure, which simulates the heat input of the solar receiver. In the discharging phase cold air is blown in the opposite direction to simulate the inlet from the process return (a pressurized air would simulate compressor outlet of an air turbine).

The objective of the test is to analyze the performance of the bed working in cycling conditions in terms of thermocline evolution until the system becomes stable (cyclic state).

Table 3. Selected cycling test data

\begin{tabular}{|l|l|}
\hline Air flow direction & $\begin{array}{l}\text { Charging (Downward) } \\
\text { Discharging (Upward) }\end{array}$ \\
\hline Air mass flow kg/s (lb/s) & $0.19(0.43)$ \\
\hline Air temperature ${ }^{\circ} \mathrm{C}\left({ }^{\circ} \mathrm{F}\right)$ & $\begin{array}{l}\text { Charging 702 (1295) } \\
\text { Discharging 146 (295) }\end{array}$ \\
\hline Air pressure & $\begin{array}{l}\text { Atmospheric pressure } \\
\text { for charging and } \\
\text { discharging }\end{array}$ \\
\hline $\begin{array}{l}\text { Initial homogeneous } \\
\text { temperature in the } \\
\text { matrix }{ }^{\circ} \mathrm{C}\left({ }^{\circ} \mathrm{F}\right)\end{array}$ & $146(295)$ \\
\hline
\end{tabular}

In the same way as for the single shot test, Figure 13 shows the experimental and the simulation results for this test. The simulation results were obtained with the model described on section 3 under the general test conditions of Table 3. For this test the experimental data for the inlet temperature of the air was not available so it was assumed constant during each phase, charge and discharge, of the cycle.

It can be appreciated in Figure 13 the very good agreement between the experimental and the simulation results for this test. The results are very similar especially in the last cycles where probably the effect of the different initial conditions applied was disappeared. 


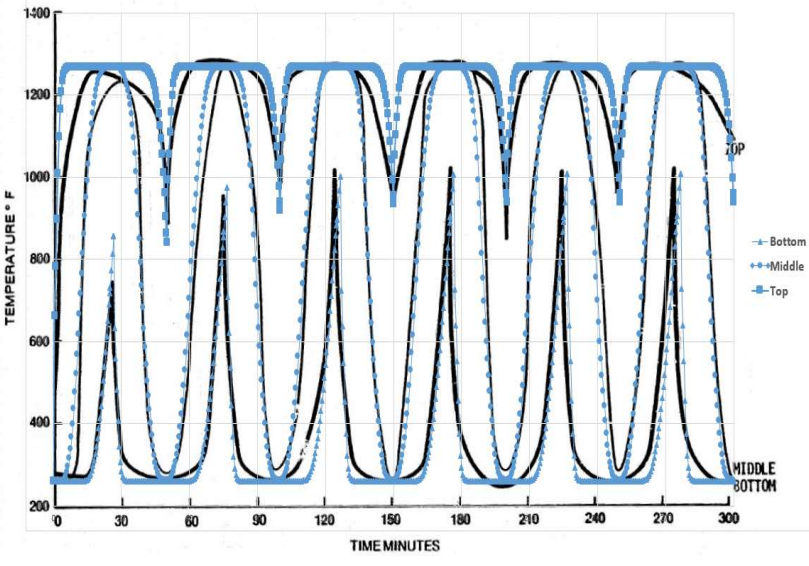

Figure 13. Experimental (black) and simulation (blue) results of cycling test of the ceramic regenerative bed model.

\section{Conclusions}

Two Modelica models have been described for the dynamic simulation of two types of regenerative beds, a metallic one based on stacked wire meshes, and a ceramic one based on straight-channelled honeycomb. These models will be used as design tool in CAPTURE project and will support the evaluation of the performance of fixed bed regenerative heat exchangers.

These models are part of a public deliverable of CAPTURE project and will be included in a free Modelica library. In addition, prototypes designed using the presented models will be tested along 2018 in the Plataforma Solar de Almería (PSA). This give the chance for validating the model with real data coming from operation of the regenerative beds.

A case study has been presented where experimental results from the testing of a ceramic honeycomb regenerator were compared with simulation results obtained with the model developed for this regenerator typology. The model provides a suitable representation of the regenerative bed behaviour and constitutes a useful tool for the design of these components.

Finally, the authors are currently working on the metallic bed models in order to study the effect of different configurations of the metallic meshes to assess the feasibility of using this type of regenerators. In addition, the metallic bed model is being extended to include other stacking configuration (in-line and staggered). These results will be presented in future publications.

\section{Acknowledgements}

The authors would like to thank the European Commission for partial funding of this work related to CAPTURE project (H2020 research and innovation programme, grant agreement No 640905).

\section{References}

R.B. Bird et all, "Transport phenomena", Wiley, New York, 1960

F. R. Casella et all. "Standardization of Thermo-Fluid Modeling in Modelica.Fluid", Proceedings of 7th International Modelica Conference, 2009, Como, Italy

S. Kakac et all, "Handbook of single-phase convective heat transfer", Wiley, New York, 1987

W.M. Kays and A.L. London, "Compact Heat Exchangers", Krieger Publishing Company, Malabar, Florida, 1998

C. Li and G.P. Peterson, "The effective thermal conductivity of wire screen", International Journal of Heat and Mass Transfer 49 (2006) 4095 -4105

W.R. Martini, "Stirling Engine Desing Manual", University Press of the Pacific, Honolulu, Hawaii, 2004

Modelica Association, "A Unified Object-Oriented Language for Physical System Modeling", 2012

K.R. Muske et all, "Model-based control of a thermal regenerator. Part 1: dynamic model", Computers and Chemical Engineering 24 (2000) 2519-2531

A. J. Organ, "The Regenerator and the Stirling Engine", Mechanical Engineering Publications Limited, London and Bury St Edmunds, UK, 1997

A. J. Organ, "Thermodynamics and Gas Dynamics of the Stirling Cycle Machine", Cambridge University Press, Cambridge, 2010

Sanders Associates Inc, Small solar electric system components demonstration final report, JPL contract 955279, Nashua, New Hampshire, August 20, 1980

A.E. Sheindlin, High temperature equipment, Hemisphere publishing Corp., Washington, 1986

J. Xu and R.A. Wirtz, "In-plane effective thermal conductivity of plain-weave screen laminates", IEEE TCPT 25 (4) (2002) 615-620 